\title{
Effects of correction equation for actual sodium concentration on the reduction in frequency of reports on pseudohyponatremia
}

https://doi.org/10.1515/labmed-2019-0058

Received March 27, 2019; accepted October 27, 2019; previously published online November 29, 2019

\section{Abstract}

Background: Serum sodium concentration could be spuriously reduced by excess glucose or lipid; thus, hyponatremia should be reassessed before reporting in order to exclude the possibility of pseudohyponatremia.

Methods: In this study, we used the calculation of corrected serum sodium concentration in hyperglycemic or lipemic samples and evaluated its effect on the reduction in frequency of reports on pseudohyponatremia. We retrospectively analyzed 1-year data from the laboratory information system.

Results: From August 2017 to July 2018, hyponatremia was initially noted in 30,465 results, of which 6614 results reported hyperglycemia. Corrected sodium concentrations were calculated using the results with glucose $>170 \mathrm{mg} / \mathrm{dL}$ or lipemia index $\geq 3$. The reported frequency of hyponatremia before and after correction of sodium concentration was reduced by $42.9 \%$. Moreover, only $51.9 \%$ of the patients with severe hyponatremia had "true" severe hyponatremia. After applying the formula, the degree of hyponatremia was unchanged in only $45.8 \%$ of the total results.

Conclusions: Our report on the corrected sodium concentration reduced the frequency of pseudohyponatremia in hyperglycemic samples. Calculation of corrected sodium

\footnotetext{
*Corresponding authors: Young Uh, MD, PhD, Department of Laboratory Medicine, Wonju Severance Christian Hospital, Yonse University Wonju College of Medicine, 20 Ilsan-ro, Wonju 26426, South Korea, Phone: +82-33-741-1592, Fax: +82-33-731-0506, E-Mail: u931018@yonsei.ac.kr; and Seong Jin Choi, MD, PhD, Department of Obstetrics and Gynecology, Wonju Severance Christian Hospital, Yonsei University Wonju College of Medicine, 20 Ilsan-ro, Wonju 26426, South Korea, Phone: +82-33-741-1278, Fax:+82-33-745-5157, E-Mail: choisj@yonsei.ac.kr Jooyoung Cho: Department of Laboratory Medicine, Yonsei University Wonju College of Medicine, Wonju, South Korea. https://orcid.org/0000-0002-9628-2334
}

concentration is a useful tool to help clinicians establish appropriate care and treatment plans. Further prospective study is required to validate to our system in a real clinical practice.

Keywords: corrected sodium; equation; hyperglycemia; hyperlipidemia; hyponatremia; pseudohyponatremia.

\section{Introduction}

Hyponatremiais the most commonelectrolyte disorder [1, 2] and a commonly encountered problem in clinical practice [3, 4]. Hyponatremia comprises $15-20 \%$ of electrolyte disorders in hospital settings [5, 6], but prompt management remains problematic because of its varying causes and conditions [4, 5, 7]. Moreover, spuriously reduced serum sodium concentration, referred to as pseudohyponatremia, is also a challenging task in both laboratory and clinical settings $[2,7,8]$. Pseudohyponatremia is not a "true" hyponatremia, and there is no hypotonicity. It can be divided into hypertonic or isotonic hyponatremia [5].

First, hyperglycemia causes hypertonic hyponatremia. Excess glucose induces diffusion of water from cells to the extracellular space and thereby causes reduced serum sodium concentration [2, 4, 9]. Kats [10] proposed that every $100 \mathrm{mg} / \mathrm{dL}$ increase in serum glucose concentration results in $1.6 \mathrm{mmol} / \mathrm{L}$ decrease in serum sodium concentration, and the conversion factor of 1.6 is still routinely used in many clinical laboratories [2].

Second, in some cases, hyponatremia is associated with artifacts in sodium concentration measurement. Presently, most automated chemical analyzers in clinical laboratories use indirect ion-selective electrode (I-ISE) methods in measuring electrolyte concentration. In the I-ISE method, the serum sample is diluted before measurement, and thereafter the apparatus measures the electrical potential across a sodium-selective membrane $[8,11]$. The I-ISE method is convenient and has both high sensitivity and specificity for the target ion it measures 
but carries some limitations [11]. The I-ISE measures the sodium concentration in aqueous samples. A normal serum sample is composed of approximately 93\% water and $7 \%$ non-aqueous components (mainly lipids and proteins) $[7,8,12]$. However, in samples with excess lipids or proteins, relatively high fractions of lipids or proteins with no sodium in themselves reduce the water fraction, thereby resulting in reporting of spurious low sodium concentration $[2,7,8]$.

Therefore, in clinical laboratories, reduced serum sodium concentration should be reassessed before reporting in order to exclude the possibility of pseudohyponatremia. In this study, we calculated the corrected serum sodium concentrations in hyperglycemic or lipemic samples submitted to our laboratory and compared them with measured concentrations.

\section{Materials and methods}

\section{Study design}

Data for this study were collected from the laboratory information system (LIS) of Wonju Severance Christian Hospital, a tertiary-level university-affiliated hospital in Wonju, South Korea. Our laboratory has reported both uncorrected (measured) and corrected sodium concentrations in hyperglycemic or lipemic samples since August 2017. Serum sodium concentrations were measured using the Roche Cobas c7000 automated chemistry analyzer (Roche Diagnostics, Rotkreuz, Switzerland). This retrospective study is based on the 1-year data (from August 2017 to July 2018) for analysis. Hyponatremia was defined as serum sodium concentration $<136 \mathrm{mmol} / \mathrm{L}[6,7]$, and hyperglycemia was defined as serum glucose concentration $>170 \mathrm{mg} / \mathrm{dL}$ [13]. Corrected sodium concentration in hyperglycemic samples was calculated according to the following equation [10]: corrected sodium concentration $(\mathrm{mmol} / \mathrm{L})=$ measured sodium concentration $(\mathrm{mmol} / \mathrm{L})+1.6 \times[$ serum glucose concentration (mg/dL)-100]/100.

Additionally, we attempted to calculate the corrected sodium concentrations in lipemic samples. Lipemia was defined as a lipemic index $\geq 3$ according to Roche Diagnostics. Corrected sodium concentrations were calculated in isotonic samples that meet all of the following conditions [3]: blood urea nitrogen (reference range, $<20 \mathrm{mg} / \mathrm{dL}$ ) and serum protein (reference range, 6.2-8.1 g/dL) concentrations are within the reference range, and serum glucose concentration is $<170 \mathrm{mg} / \mathrm{dL}$. Corrected sodium concentration in lipemia was calculated according to the following equation $[7,8]$ : corrected sodium concentration in $\mathrm{mmol} / \mathrm{L}=$ (measured sodium concentration in $\mathrm{mmol} / \mathrm{L} \times 0.93) /[0.991-(0.001 \times$ serum triglyceride concentration in $\mathrm{mg} / \mathrm{dL})-(0.007 \times$ serum protein concentration in $\mathrm{g} / \mathrm{dL}$ )].

This study was approved by the institutional review board (IRB) of Wonju Severance Christian Hospital (IRB no. CR318071), which waived the requirement for informed consent.

\section{Statistical analysis}

The degree of hyponatremia according to sodium concentrations was divided into subgroups as severe (sodium concentration $<126 \mathrm{mmol} / \mathrm{L})$, moderate (126-130 mmol/L), mild hyponatremia (131-135 mmol/L), and normonatremia ( $\geq 136 \mathrm{mmol} / \mathrm{L}$ ). Moreover, the degree of hyperglycemia according to serum glucose concentrations was divided into subgroups as group I (serum glucose concentration of $171-200 \mathrm{mg} / \mathrm{dL})$, group II (201-300 mg/dL), group III (301-400 mg/dL), group IV (401-600 mg/dL), and group $\mathrm{V}(>600 \mathrm{mg} / \mathrm{dL})$. We compared the frequency of reporting hyponatremia before and after correction of sodium concentrations for each subgroup and total result. Deltas of sodium concentrations (corrected sodium - initial sodium) were also analyzed and compared.

Statistical analysis was performed using SPSS version 23.0 (IBM Corp., Armonk, NY, USA) and Microsoft Excel 2016 (Microsoft Corp, Redmond, WA, USA) with Analyse-it version 5.01 (Analyse-it Software, Ltd., Leeds, UK).

\section{Results}

A total of 252,892 serum sodium results were reported during the study period, and hyponatremia was found in $30,465(12.0 \%)$ results. Of these, hyperglycemia was found in 6614 results. Of the results reporting both hyponatremia and hyperglycemia, before correction of sodium concentrations, the proportions of degree of hyponatremia were as follows: $322(4.9 \%)$ in the severe hyponatremia subgroup, $1136(17.2 \%)$ in the moderate hyponatremia subgroup, and $5156(78.0 \%)$ in the mild hyponatremia subgroup. Furthermore, the proportions of degree of hyperglycemia were as follows: 2017 (30.5\%) in group I, 3053 (46.2\%) in group II, $927(14.0 \%)$ in group III, 455 (6.9\%) in group IV, and 162 (2.4\%) in group V (Table 1$)$.

After correction of sodium concentrations using the formula for hyperglycemia, the overall frequency of 
Table 1: Distribution of initial serum sodium concentrations before correction.

\begin{tabular}{|c|c|c|c|c|}
\hline \multirow{2}{*}{$\begin{array}{l}\text { Degree of hyperglycemia according to } \\
\text { serum glucose concentration, } \mathrm{mg} / \mathrm{dL}\end{array}$} & \multicolumn{4}{|c|}{ No. (\%) of each subgroup by sodium concentration ( $\mathrm{mmol} / \mathrm{L}$ ) before correction } \\
\hline & Severe $(<126)$ & Moderate (126-130) & Mild (131-135) & Total \\
\hline Group I (171-200) & $77(3.8)$ & $328(16.3)$ & $1612(79.9)$ & $2017(30.5)$ \\
\hline Group II (201-300) & $125(4.1)$ & $490(16.0)$ & $2438(79.9)$ & $3053(46.2)$ \\
\hline Group III (301-400) & $45(4.9)$ & $157(16.9)$ & $725(78.2)$ & $927(14.0)$ \\
\hline Group IV (401-600) & $34(7.5)$ & $95(20.9)$ & $326(71.6)$ & $455(6.9)$ \\
\hline Group V $(>600)$ & $41(25.3)$ & $66(40.7)$ & $55(34.0)$ & $162(2.4)$ \\
\hline Total & $322(4.9)$ & $1136(17.2)$ & $5156(77.9)$ & $6614(100)$ \\
\hline
\end{tabular}

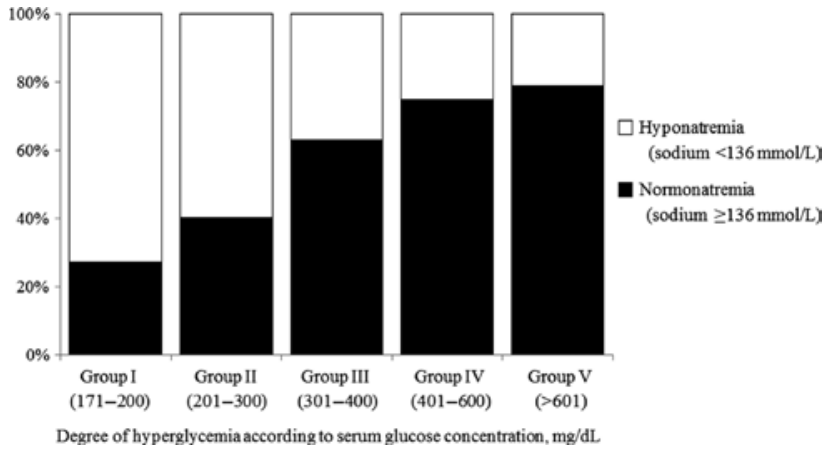

Figure 1: Reported frequency of hyponatremia after correction of sodium concentrations.

reports on hyponatremia was reduced by 2838 (42.9\%). Only 3776 results (57.1\%) still showed hyponatremia after calculation of corrected sodium concentrations. Reduced frequency of hyponatremia after correction of sodium concentrations were as follows: $551(27.3 \%)$ in group I, 1232 (40.4\%) in group II, 586 (63.2\%) in group III, 341 (74.9\%) in group IV, and $128(79.0 \%)$ in group V (p for trend $<0.001$ ) (Figure 1). The proportions of degree of hyponatremia after correction of sodium concentrations were as follows: $167(2.5 \%)$ in the severe hyponatremia subgroup, 568 (8.6\%) in the moderate hyponatremia subgroup, and 3041 $(46.0 \%)$ in the mild hyponatremia subgroup (Table 2).
After applying the formula for hyperglycemia, the percentages of results of which the sodium concentration was changed into normonatremia results after correction from mild, moderate, and severe hyponatremia results were $53.3 \%, 6.9 \%$, and $4.3 \%$, respectively (Table 3 ). The degree of hyponatremia was changed in $34.5 \%$ in group I, 50.8\% in group II, $79.0 \%$ in group III, $98.0 \%$ in group IV, and 98.1\% in group V (Table 4). Moreover, the median values for delta of sodium concentrations for each subgroup was 1.3 (interquartile range [IQR], 1.2-1.4) in group I, 2.1 (IQR, 1.8-2.5) in group II, 3.7 (IQR, 3.4-4.1) in group III, 5.6 (IQR, 5.2-6.5) in group IV, and 9.6 (IQR, 8.8-11.6) in group V (p for trend $<0.001$ ) (data not shown).

Additionally, we have attempted to calculate corrected sodium concentrations in lipemic samples. During this period, only 68 results had lipemic index $\geq 3$ according to Roche Diagnostics. Of the 68 samples, sodium concentrations were $<136 \mathrm{mmol} / \mathrm{L}$ only in $11(16.2 \%)$ samples: 10 results with mild hyponatremia and only one result with moderate hyponatremia. Of 11 results, three $(27.3 \%)$ with hyponatremia before correction were changed into normonatremia after correction. The mean delta sodium concentration of 11 results was $1.3 \mathrm{mmol} / \mathrm{L}$. When compared with the sodium concentrations on blood gas analysis (five of 11 results), the mean difference was $1.6 \mathrm{mmol} / \mathrm{L}$.

Table 2: Distribution of sodium concentrations after correction.

\begin{tabular}{|c|c|c|c|c|c|}
\hline \multirow{2}{*}{$\begin{array}{l}\text { Degree of hyperglycemia } \\
\text { according to serum glucose } \\
\text { concentrations, } \mathrm{mg} / \mathrm{dL}\end{array}$} & \multicolumn{5}{|c|}{ No. (\%) of each subgroup by serum sodium concentration (mmol/L) after correction } \\
\hline & Severe $(<126)$ & Moderate (126-130) & Mild (131-135) & Normonatremia $(>136)$ & Total \\
\hline Group I (171-200) & $55(2.7)$ & $227(11.3)$ & $1184(58.7)$ & $551(27.3)$ & $2017(30.5)$ \\
\hline Group II (201-300) & $82(2.7)$ & $256(8.4)$ & $1483(48.6)$ & $1232(40.3)$ & $3053(46.2)$ \\
\hline Group III (301-400) & $22(2.4)$ & $57(6.1)$ & $262(28.3)$ & $586(63.2)$ & $927(14.0)$ \\
\hline Group IV (401-600) & $5(1.1)$ & $22(4.8)$ & 87 (19.1) & $341(75.0)$ & $455(6.9)$ \\
\hline Group V (>600) & $3(1.9)$ & $6(3.7)$ & $25(15.4)$ & $128(79.0)$ & $162(2.4)$ \\
\hline Total & $167(2.5)$ & $568(8.6)$ & $3041(46.0)$ & $2838(42.9)$ & $6614(100)$ \\
\hline
\end{tabular}


Table 3: Comparison of the pairwise results for degree of hyponatremia between sodium concentrations before and after correction.

\begin{tabular}{|c|c|c|c|c|c|}
\hline \multirow{2}{*}{$\begin{array}{l}\text { No. (\%) of each subgroup } \\
\text { before correction }\end{array}$} & \multicolumn{5}{|c|}{ No. (\%) of each subgroup by serum sodium concentration ( $\mathrm{mmol} / \mathrm{L}$ ) after correction } \\
\hline & Severe $(<126)$ & Moderate (126-130) & Mild (131-135) & Normonatremia $(>136)$ & Total \\
\hline Severe $(<126)$ & $167(51.9)$ & $116(36.0)$ & $25(7.8)$ & $14(4.3)$ & $322(4.9)$ \\
\hline Moderate (126-130) & - & $452(39.8)$ & $606(53.3)$ & $78(6.9)$ & $1136(17.2)$ \\
\hline Mild (131-135) & - & - & $2410(46.7)$ & $2746(53.3)$ & $5156(77.9)$ \\
\hline Total & $119(2.5)$ & $568(8.6)$ & $3041(46.0)$ & 2838 (42.9) & $6614(100)$ \\
\hline
\end{tabular}

Table 4: Comparison of the pairwise results for degree of hyponatremia between sodium concentrations before and after correction.

\begin{tabular}{|c|c|c|c|c|c|}
\hline \multirow{2}{*}{$\begin{array}{l}\text { Degree of hyperglycemia } \\
\text { according to serum glucose } \\
\text { concentration, } \mathrm{mg} / \mathrm{dL}\end{array}$} & \multicolumn{5}{|c|}{ No. (\%) of grading difference between sodium concentrations before and after correction } \\
\hline & 0 & 1 & 2 & 3 & Total \\
\hline Group I (171-200) & $1321(65.5)$ & $696(34.5)$ & - & - & $2017(30.5)$ \\
\hline Group II (201-300) & $1501(49.2)$ & $1552(50.8)$ & - & - & $3053(46.2)$ \\
\hline Group III (301-400) & $195(21.0)$ & $732(79.0)$ & - & - & $927(14.0)$ \\
\hline Group IV (401-600) & $9(2.0)$ & $420(92.3)$ & $26(5.7)$ & - & $455(6.9)$ \\
\hline Group V $(>600)$ & $3(1.9)$ & $68(42.0)$ & $77(47.5)$ & $14(8.6)$ & $162(2.4)$ \\
\hline Total & $3029(45.8)$ & $3468(52.4)$ & $103(1.6)$ & $14(0.2)$ & $6614(100)$ \\
\hline
\end{tabular}

\section{Discussion}

Hyponatremia is common in clinical practice. The causes of hyponatremia vary from mild disorder to systemic problem [14]. Typically, the main reason of hyponatremia is abnormal retention of water, such as excess intake or infusion of water, which leads to a hypotonic status [2]. In patients with mild symptoms, hyponatremia with signs and symptoms (e.g. nausea, vomiting, dizziness, gait disturbance) needs a proper approach including fluid restriction or vasopressin antagonist administration. And, some cases of acute hyponatremia require medical intervention, such as the administration of $3 \%$ sodium chloride, depending on the unique clinical circumstances [3]. However, the approach to patients with hyponatremia should be performed with caution. Rapid or inappropriate correction of sodium concentration carries the risk of osmotic demyelination syndrome due to re-accumulation of osmolytes in the cells [5]. On the other hand, patients with pseudohyponatremia usually exhibit normal osmolar pressure, which, if mistaken for hypo-osmolar hyponatremia and given fluid restriction, is feared to further exacerbate the level of hyperviscosity and hypercoagulability [15]. Pseudohyponatremia is a term that reflects the analytical artifact for measuring sodium level and should be corrected by a formula that reflects the actual level in order to help clinicians determine the patient's condition correctly [16].
As mentioned, serum sodium concentration is influenced by some factors. Hyperglycemia induces hyponatremia by an extracellular shift of water [8]; however, previous studies have shown that decrease in serum glucose concentration in patients with hyperglycemia results in increased serum sodium concentration due to return of water to the intercellular space [5, 9]. Thus, correction of serum sodium concentration by sodium glucose concentration correction is recommended in patients with hyperglycemia $[4,10]$. Furthermore, hyperlipidemia and hyperproteinemia can also induce hyponatremia in samples measured using the I-ISE method. The direct ISE method can overcome this problem and measure the actual sodium concentration, but the I-ISE method has been widely used in routine clinical laboratories. Because of relatively lower proportion of water fraction in samples that contain excess lipid or protein, an I-ISE-based analyzer has a tendency to report pseudohyponatremia. Thus, actual serum water content should be calculated in hyperlipidemia or hyperproteinemia in order to avoid misclassification of hyponatremia.

In our laboratory, we have reported both uncorrected (measured) and corrected sodium concentrations when measured serum sodium concentration is $<136 \mathrm{mmol} / \mathrm{L}$ in hyperglycemic or lipemic samples since August 2017. Certainly, there is a concern about the reliability and usefulness of the corrected sodium concentration. However, this change was implemented by our laboratory to help 
clinicians interpret their results with hyperglycemia or hyperlipidemia. We present both pre- and postcorrection concentrations and make them available for use in clinical settings. We retrospectively collected 1-year data on corrected sodium concentrations and evaluated the effects of the calculation on reduction of frequency of reports on pseudohyponatremia. During the 1-year period, reports on pseudohyponatremia were reduced by $42.9 \%$ of the total results with hyponatremia. Of the results with mild hyperglycemia (serum glucose concentration, $171-200 \mathrm{mg} / \mathrm{dL}$ ) and severe hyperglycemia ( $>600 \mathrm{mg} / \mathrm{dL}$ ), $27.3 \%$ and $79.0 \%$, respectively, were confirmed to be pseudohyponatremia. Moreover, of the results with severe hyponatremia (serum sodium concentration $<121 \mathrm{mmol} / \mathrm{L}$ ), only $51.9 \%$ were "true" severe hyponatremia. After applying the formula, the degree of hyponatremia was unchanged only in 3029 (45.8\%) results from patients with hyperglycemia. The percentage of one grading difference was $52.4 \%$. Furthermore, even in samples with severe hyperglycemia, three grading differences were observed in 14 samples. The trend of delta sodium concentrations was similar to that of other results in this study.

This study has some limitations. First, we used a correction factor of $1.6 \mathrm{mmol} / \mathrm{L}$ in calculating corrected sodium concentrations according to Kats' proposal [10]. However, Hiller [9] suggested a correction factor of $2.4 \mathrm{mmol} / \mathrm{L}$. After applying Hiller's formula, reduced frequency of hyponatremia was as follows: $742(36.8 \%)$ in group I, 1764 (57.8\%) in group II, 742 (80.0\%) in group III, $397(87.3 \%)$ in group IV, and $153(94.4 \%)$ in group V (p for trend $<0.001$ ) (data not shown). Compared with those in Kats' formula, reduced frequency of hyponatremia was statistically significantly lower in all groups in Hiller's formula ( $\mathrm{p}<0.001$ for all groups). The hyponatremic effect of hyperglycemia has been analyzed in several studies, but the accuracy of each correction factor has not been proven yet. This is one of limitations of our study, and if a more reliable correction factor is proven, we will change the formula applied to our LIS.

Second, we did not assess clinical outcomes in this study. In fact, this study was waived the requirement for informed consent by the IRB on the condition it uses only patients' laboratory data without using the patients' personal information or medical chart review. For these reasons, we could not reflect the clinical courses or outcomes. In this study, as we present both pre- and postcorrection data, it is expected that clinicians will be able to use our data to make judgments if there is a discrepancy between the sodium level and actual clinical status of the patient with hyponatremia. However, we did not include a survey of how clinicians feel and what points should be improved to our system. Therefore, further study is needed to reflect this clinical aspect.

Additionally, we have attempted to calculate corrected sodium concentrations in lipemic samples. However, as mentioned, only 11 results had both hyponatremia and hyperlipidemia. Of these, three samples had a high triglyceride concentration ( $>2000 \mathrm{mg} / \mathrm{dL}$ ), which would affect our formula. Moreover, hyperproteinemia was not found in any samples. Moreover, the number of samples was small, so statistically significant results were not obtained in hyperlipidemia. This is also one of limitations in our study. Further studies are required to evaluate the efficacy of the formula for hyperlipidemia on reduction of frequency of reports on pseudohyponatremia.

In conclusion, our laboratory has applied the formula of corrected sodium concentration for hyperglycemia or hyperlipidemia since August 2017. The report on corrected sodium concentration has reduced the frequency of pseudohyponatremia in hyperglycemic samples. Calculation of corrected sodium concentration is a useful tool to help clinicians establish appropriate care and treatment plans. Further prospective study is required to validate to our system in a real clinical practice.

Author contributions: All the authors have accepted responsibility for the entire content of this submitted manuscript and approved submission.

Research funding: None declared.

Employment or leadership: None declared.

Honorarium: None declared.

Competing interests: The funding organization(s) played no role in the study design; in the collection, analysis, and interpretation of data; in the writing of the report; or in the decision to submit the report for publication.

\section{References}

1. Liamis G, Rodenburg EM, Hofman A, Zietse R, Stricker BH, Hoorn EJ. Electrolyte disorders in community subjects: prevalence and risk factors. Am J Med 2013;126:256-63.

2. Oh MS, Briefel G. Evaluation of renal function, water, electrolytes, and acid-base balance. In: McPherson RA, Pincus MR, editor. Henry's clinical diagnosis and management by laboratory methods. St. Louis, MO: Elsevier Saunders, 2017:162-87.

3. Sahay M, Sahay R. Hyponatremia: a practical approach. Indian J Endocrinol Metab 2014;18:760-71.

4. Wolf MB. Hyperglycemia-induced hyponatremia: reevaluation of the $\mathrm{Na}+$ correction factor. J Crit Care 2017;42:54-8.

5. Spasovski G, Vanholder R, Allolio B, Annane D, Ball S, Bichet D, et al. Clinical practice guideline on diagnosis and treatment of hyponatraemia. Eur J Endocrinol 2014;170:G1-47. 
6. Lee JJ, Kilonzo K, Nistico A, Yeates K. Management of hyponatremia. Can Med Assoc J 2014;186:E281-6.

7. Liamis G, Liberopoulos E, Barkas F, Elisaf M. Spurious electrolyte disorders: a diagnostic challenge for clinicians. Am J Nephrol 2013;38:50-7.

8. Weisberg LS. Pseudohyponatremia: a reappraisal. Am J Med 1989;86:315-8.

9. Hillier TA, Abbott RD, Barrett EJ. Hyponatremia: evaluating the correction factor for hyperglycemia. Am J Med 1999;106:399-403.

10. Katz MA. Hyperglycemia-induced hyponatremia-calculation of expected serum sodium depression. $\mathrm{N}$ Engl J Med 1973;289:843-4.

11. Pincus MR, Lifshitz MS, Bock JL. Analysis: principles of instrumentation. In: McPherson RA, Pincus MR, editors. Henry's clinical diagnosis and management by laboratory methods. St. Louis, MO: Elsevier Saunders, 2017:33-59.

12. Nguyen MK, Ornekian V, Butch AW, Kurtz I. A new method for determining plasma water content: application in pseudohyponatremia. Am J Physiol Renal Physiol 2007;292:F1652-6.

13. Duncan AE. Hyperglycemia and perioperative glucose management. Curr Pharm Des 2012;18:6195-203.

14. Yeates KE, Singer M, Morton AR. Salt and water: a simple approach to hyponatremia. Can Med Assoc J 2004;170:365-9.

15. Turchin A, Seifter JL, Seely EW. Mind the gap. N Engl J Med 2003;349:1465-9.

16. Lippi G, Aloe R. Hyponatremia and pseudohyponatremia: first, do no harm. Am J Med 2010;123:e17. 